\title{
Brain Abscess in a Patient With Radiotherapy-Treated Adenoid Cystic Carcinoma: A Misdiagnosis Case Report and Review of the Literature
}

\author{
Christopher Macko ${ }^{a}$, Sophia Ahmed ${ }^{\text {b, d }}$, Ali Seific
}

\begin{abstract}
Brain abscesses are a relatively rare entity with an estimated incidence of 0.3 to 1.3 per 100,000 people per year. Brain abscesses arise from direct contiguous spread, hematogenous spread, neurosurgical procedures, open traumatic brain injuries, and cryptogenic sources. Early identification is pivotal, as delayed diagnosis and treatment lead to a very poor prognosis. Our case illustrates an elderly gentleman with a history of adenoid cystic carcinoma (ACC) of the oropharyngeal palate who presented to an outside hospital with severe headaches and was found to have a questionable metastatic lesion to his left temporal region. He was discharged with a course of steroids. Weeks later his headaches persisted, mentation further declined and repeat imaging revealed the same abnormal lesion. He subsequently underwent a craniotomy and was found to have a significant temporal abscess and empyema, which were evacuated. Post-operatively his course was complicated by status epilepticus requiring intubation and he was ultimately placed on hospice care. Our case illustrates the importance of early recognition and intervention for suspicious lesions, particularly when predisposing risk factors exist.
\end{abstract}

Keywords: Brain abscess; Adenoid cystic carcinoma; Radiotherapy

\section{Introduction}

Brain abscesses are a relatively rare entity yet have devastating effects when left untreated. Brain abscesses arise from direct

Manuscript submitted July 26, 2020, accepted August 3, 2020

Published online August 14, 2020

aSchool of Medicine, University of Texas Health Science Center at San Antonio, San Antonio, TX 78229, USA

bDepartment of Emergency Medicine, University of Texas Health Science Center at San Antonio, San Antonio, TX 78229, USA

'Department of Neurosurgery, University of Texas Health Science Center at San Antonio, San Antonio, TX 78229, USA

${ }^{\mathrm{d} C}$ Corresponding Author: Sophia Ahmed, Department of Emergency Medicine, University of Texas Health Science Center at San Antonio, 7703 Floyd Curl Dr, MC 7736, San Antonio, TX 78229, USA. Email: Ahmeds1@uthscsa.edu

doi: https://doi.org/10.14740/jnr620 contiguous spread, hematogenous spread, neurosurgical procedures, open traumatic brain injuries, and cryptogenic sources [1, 2]. Brain abscesses from hematogenous spread usually involve the distribution of the middle cerebral artery and are found in the region of the grey white junction [2]. Direct spread is dependent on the origin of the original infection. Infection of the paranasal sinuses usually track to the frontal lobes whereas temporal lobe abscesses typically arise from otitis or mastoiditis [2].

Here we present a patient with a brain abscess who had previous history of adenoid cystic carcinoma (ACC) of the oropharyngeal palate. He presented with altered mental status that was misdiagnosed as ACC metastasis to the brain.

\section{Case Report}

A 68-year-old man with a past medical history of metastatic ACC of the oropharyngeal palate presented with a 2-week history of lethargy, confusion and headaches. The patient had visited an outside hospital (OSH) 1-week prior with similar symptoms. At the OSH, he was found to have a left anterior temporal lesion that was presumed to be brain metastasis and was discharged on dexamethasone. His condition improved for 1 week, but then deteriorated.

On review of the patient's cancer history, we learned that he had been diagnosed with ACC in December of 2015 and underwent chemo-radiation in December 2015 - March 2016. The cancer recurred and eventually required a left maxillectomy with pterygopalatine fossa dissection in 2019 and subsequent radiation (3500 cGy) due to positive margins.

On admission to our facility he had a Glasgow Coma Scale (GCS) of Eye (E) 4-Verbal (V) 4-Motor (M) 6 with no focal neurological deficits. Initial laboratory studies were within normal limits except for a white blood cell count of $35 \times 10^{9}$ cells $/ \mathrm{L}$ and a sodium of $130 \mathrm{mEq} / \mathrm{L}$. Head computed tomography (CT) at that time showed vasogenic edema within the left temporal lobe (Fig. 1a), $1 \mathrm{~cm}$ midline shift, an early uncal herniation, and a $7 \mathrm{~mm}$ subdural hematoma over the left cerebral convexity. It also showed chronic sphenoid sinusitis with surrounding osseous remodeling.

The patient was started on hypertonic saline for the vasogenic edema, loaded with levetiracetam and placed on continuous electroencephalogram (cEEG) to rule out subclinical seizures as a cause of his altered mental status. His exam sub- 


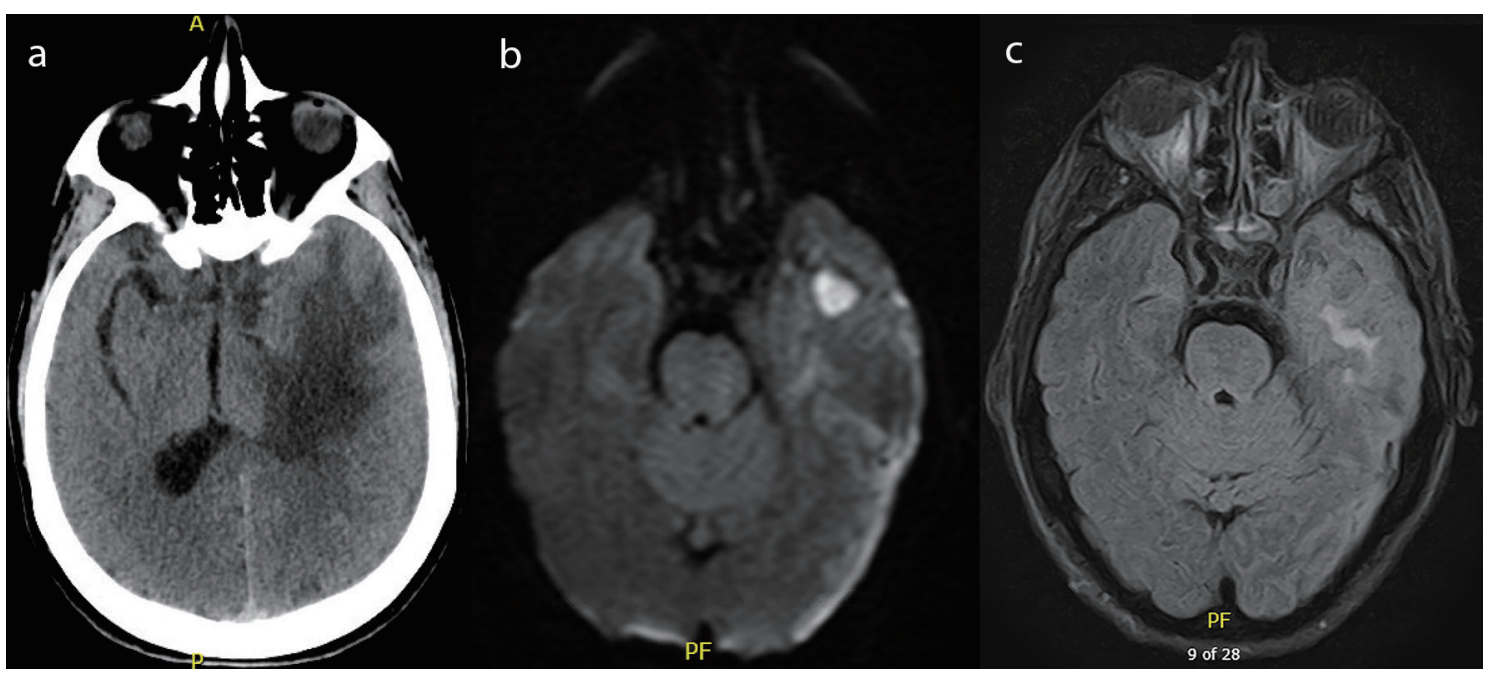

Figure 1. (a) Computed tomography of the head with and without contrast demonstrating left temporal lobe vasogenic edema. (b) Magnetic resonance imaging of the brain with and without contrast, diffusion weighted imaging demonstrating a well-circumscribed lesion to the left temporal lobe. (c) Magnetic resonance imaging of the brain with and without contrast, flair images demonstrating vasogenic edema to the left temporal lobe.

sequently declined to a GCS of E4-V1-M5 with localization to all extremities. A decision was made with the family to perform an anterior temporal lobectomy to prevent herniation. Prior to surgery, a magnetic resonance imaging (MRI) scan of his brain was obtained which showed a left temporal lobe $2.1 \mathrm{~cm}$ rim enhancing collection with diffusion restriction, concerning for an abscess (Fig. 1b, c). Additionally, it showed a left cerebral convexity concerning for a subdural empyema and an increasing midline shift of $1.2 \mathrm{~cm}$ associated with subfalcine and uncal herniation. It also showed focal dehiscence of the sphenoid sinus wall in communication with the middle cranial fossa.

The patient underwent a left craniotomy and was found to have a thick compressive subdural empyema and left temporal lobe intraparenchymal abscess. The surgeons evacuated the subdural empyema (about $100 \mathrm{~cm}^{3}$ ) and left temporal lobe abscess. Cultures were sent from the abscess and the patient was started on vancomycin, cefepime, and metronidazole for empirical coverage. He was extubated post-operatively, however his mentation did not improve. No seizures were recorded thus far, but the patient remained on cEEG due to concern for seizures post-operatively (Fig. 2).

The following day the patient's exam was unchanged, and he was noted to be in non-convulsive status epilepticus. He was loaded with fosphenytoin and started on maintenance phenytoin. He also received lorazepam, lacosamide and his dose of levetiracetam was increased. He continued to seize despite the anti-epileptic regimen, so he was intubated and placed on midazolam and propofol drips. He was adequately burst suppressed and ultimately weaned off both midazolam and propofol, with no improvement in his mentation or change in clinical exam.

Otolaryngology (ENT) was consulted and they strongly suspected an otitis media as a source of the infection and performed a left wide field myringotomy. They also took cultures from the patient's sinuses and oropharyngeal wall. Cultures from the temporal lobe abscess, subdural empyema, blood and oropharyngeal wall all grew out streptococcus intermedius.
The patient's mentation and clinical exam did not improve despite burst suppression and removal of all sedative medications. A family meeting was held in combination with ENT, neurosurgery, and neurocritical care and the family ultimately decided to place the patient in hospice care.

\section{Discussion}

Our case is a unique presentation where a patient with $\mathrm{ACC}$ of the oropharyngeal palate treated with radiation developed a temporal lobe brain abscess. To the best of our knowledge this is likely the first reported case of this particular constellation of radiotherapy-treated ACC leading to a brain abscess. Upon review of the literature, we discovered that this pattern of a radiotherapy-treated head and neck cancer developing a brain abscess is rare, but more commonly seen in radiotherapy-treated nasopharyngeal carcinoma (NPC) patients [3-5]. It is thought

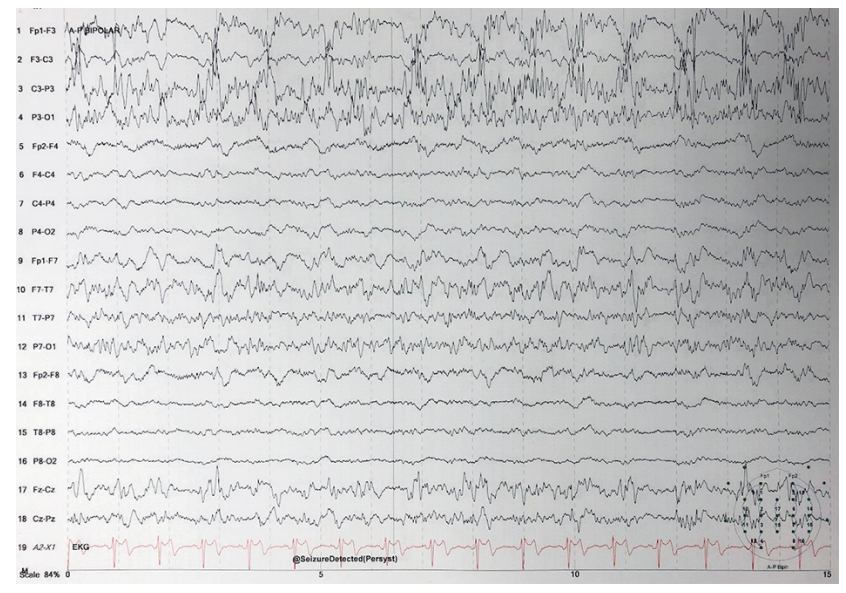

Figure 2. Electroencephalogram demonstrating status epilepticus. 
Table 1. Summary of Retrospective Studies, Case Series, and Case Reports Related to Abscesses Arising After Radiation Therapy

\begin{tabular}{|c|c|c|}
\hline Authors & Type of study & Key findings \\
\hline $\begin{array}{l}\text { Chuang et } \\
\text { al, } 2015[3]\end{array}$ & $\begin{array}{l}\text { Retrospective study of } 146 \text { patients } \\
\text { with brain abscesses ( } 10 \text { of whom } \\
\text { had received radiotherapy for a head } \\
\text { and neck cancer). }\end{array}$ & $\begin{array}{l}\text { The pathogenesis of bacterial brain abscess in post-irradiated NPC patients is } \\
\text { likely due to radiation and the tumor both destroyed the skull base and resulted in } \\
\text { a breakdown of the blood-brain barrier, providing a pathway for the pathogens; } \\
\text { increase in the incidence of chronic otitis media, sphenoid sinusitis, or other nasal } \\
\text { infection. Radiation-related temporal lobe necrosis is also a predisposing factor. }\end{array}$ \\
\hline $\begin{array}{l}\text { Fang et al, } \\
2012[4]\end{array}$ & $\begin{array}{l}\text { Retrospective analysis of } 210 \text { patients } \\
\text { with brain abscesses, } 12 \text { of whom } \\
\text { had radiotherapy-treated NPC. }\end{array}$ & $\begin{array}{l}\text { NPC patients post radiotherapy with brain abscesses frequently had chronic otitis } \\
\text { media; temporal lobe as the most common site; high incidence of post-radiation } \\
\text { necrosis; staphylococcus and streptococcus as the most common species. }\end{array}$ \\
\hline $\begin{array}{l}\text { Liang et al, } \\
2009[5]\end{array}$ & $\begin{array}{l}\text { Retrospective study of } 18 \text { post- } \\
\text { irradiated NPC patients with CNS } \\
\text { infection (brain abscess, cavernous } \\
\text { sinus thrombosis, epidural abscess, } \\
\text { and meningitis). }\end{array}$ & $\begin{array}{l}\text { Post-irradiated NPC patients with skull base osteoradionecrosis are prone to have } \\
\text { CNS infections. The prevalence of otitis media and rhinosinusitis is high in post- } \\
\text { irradiated NPC patients. Ten patients with CNS infection had skull base } \\
\text { osteoradionecrosis. }\end{array}$ \\
\hline $\begin{array}{l}\text { Kuriyama et } \\
\text { al, } 2019 \text { [9] }\end{array}$ & $\begin{array}{l}\text { Case report of a patient with scalp } \\
\text { angiosarcoma and a subsequent brain } \\
\text { abscess after treatment. }\end{array}$ & $\begin{array}{l}\text { Brain abscess formation in this patient could be due to large skin defects and cranial } \\
\text { bone necrosis resulting from surgery and radiation. }\end{array}$ \\
\hline $\begin{array}{l}\text { Noguchi et } \\
\text { al, } 2018[10]\end{array}$ & $\begin{array}{l}\text { Case report of oropharyngeal } \\
\text { carcinoma treated with radiotherapy } \\
\text { and with a subsequent brain abscess } \\
\text { several years later. }\end{array}$ & $\begin{array}{l}\text { Patient underwent chemoradiotherapy for oropharyngeal carcinoma. Was later found } \\
\text { to have brain abscess that was presumed to be caused by radiation osteomyelitis of } \\
\text { the mandible. }\end{array}$ \\
\hline
\end{tabular}

NPC: nasopharyngeal cancer; CNS: central nervous system.

that the radiation and direct effects from the tumors predispose these patients to brain abscesses as many of these patients have chronic nasal and middle ear infections, skull base deficits, and radiation necrosis of the brain [3-5]. We found that these same predisposing conditions existed in our patient.

We searched Medline using the key words, "brain abscess radiation" and searched the 121 results for relevant articles. We found three retrospective studies, one case series, and two case reports that document and evaluate this association. We also found mentions of this association in at least two other articles $[6,7]$. The retrospective studies, case series, and case reports are summarized in Table 1 [3-5, 8-10].

The majority of the research in this field has been done in patients with NPC, as radiotherapy is the mainstay of treatment for patients with NPC [4]. The true prevalence of brain abscesses post-radiotherapy for NPC or other head and neck cancers is not known, however this association has been documented in the literature on multiple occasions (Table 1).

On review of the studies above we were able to identify three main risk factors. First, skull base deficits as either the result of osteoradionecrosis or direct invasion of bone by the tumor disrupts the natural barrier between the central nervous system and areas such as the nasopharynx, sinuses, and middle ear $[3,5,8]$. Secondly, histories of sinusitis and chronic otitis media are common in patients treated with radiotherapy for NPC $[3-5,8]$. The exact pathogenesis is unclear, but it is thought that the tumor itself and/or radiotherapy lead to eustachian tube dysfunction, thus predisposing them to sinusitis and otitis [4]. Finally, radionecrosis of the brain places brain tissue at an increased risk for infection $[3,4,8]$. Coagulation and fibrinoid necrosis of small blood vessels as a direct result of radiotherapy, leads to parenchymal ischemia and necrosis. This now infarcted brain tissue shows a greater tendency to develop abscesses compared to normal brain tissue [2, 3]. Radionecrosis typically develops 2 years after radiotherapy [11].

It is unclear exactly how our patient developed his brain abscess, although he shares many of the risk factors that have been identified in other patients with brain abscesses post-radiotherapy. On our patient's numerous brain imaging studies, he was found to have nonspecific soft tissue attenuation of the left external auditory canal with possible middle ear involvement, a left mastoid air cell effusion, and acute on chronic sphenoid sinusitis with a dehiscent sphenoid sinus wall. Furthermore, on his MRI brain 3 months prior to admission he was found to have findings consistent with radiation necrosis. The patient's family also reported a history of left ear pain for several months prior to his admission.

Based on these imaging results, a possible pathogenesis could be direct seeding of the temporal lobe through a chronic left sphenoid sinusitis in the presence of a dehiscent sphenoid sinus wall and a predisposition for infection of the temporal lobe due to radiation necrosis. It is also possible that there was direct seeding of the infection either from middle ear infection or subsequent mastoiditis. Brain abscesses from sphenoid sinuses infections classically track to the frontal lobes and temporal lobe abscesses classically result from mastoiditis and or middle ear infections [2]. However, it is possible with necrosis of the sphe- 
noid bone for there to be contiguous spread to the anterior temporal lobes, thus we cannot rule out the sphenoid sinus as our source for infection [2]. Streptococci are common organisms for brain abscesses from both sinus and otogenic sources and thus cannot be used to differentiate the source of infection.

Detection of brain abscesses is challenging in radiotherapy-treated head and neck cancer patients because differentiating metastasis, abscess, and radionecrosis can be difficult. All can present as ring-enhancing masses with edema on CT or MRI [11]. This complicated the patient's course as the OSH initial evaluation strongly suspected metastasis as the cause for our patient's temporal lesion and the patient was started on dexamethasone instead of empiric broad-spectrum antibiotics.

Post-radiotherapy brain abscesses are a well-described complication in NPC patients, but are less well described in other head and neck cancers. While not classically listed as a risk factor, brain abscesses should be considered in post-radiotherapy head and neck cancer patients presenting with new brain lesions on imaging. Early identification and treatment can help prevent the devastating outcome of delayed diagnosis and treatment.

\section{Acknowledgments}

None to declare.

\section{Financial Disclosure}

We have no financial or funding disclosures.

\section{Conflict of Interest}

We have no conflicts of interest financial or otherwise.

\section{Informed Consent}

Informed consent was not obtained from the patient as the patient went on hospice and there are no identifying features in the paper.

\section{Author Contributions}

All authors contributed equally to the production of this manuscript. The entire manuscript was reviewed and edited by all individuals listed.

\section{Data Availability}

The authors declare that data supporting the findings of this study are available within the article. All data was extrapolated from referenced studies and those studies may be reviewed for specific details pertaining to their data collection.

\section{References}

1. Brouwer MC, Coutinho JM, van de Beek D. Clinical characteristics and outcome of brain abscess: systematic review and meta-analysis. Neurology. 2014;82(9):806813.

2. Jameson JL, Kasper DL, Fauci AS, et al. Harrison's Principles of Internal Medicine, 20e. New York, NY: McGraw-Hill Education; 2018.

3. Chuang JM, Lin WC, Fang FM, Huang YJ, Ho JT, Lu $\mathrm{CH}$. Bacterial brain abscess formation in post-irradiated patients: What is the possible pathogenesis? Clin Neurol Neurosurg. 2015;136:132-138.

4. Fang $\mathrm{PH}$, Lin WC, Tsai NW, Chang WN, Huang CR, Chang HW, Huang TL, et al. Bacterial brain abscess in patients with nasopharyngeal carcinoma following radiotherapy: microbiology, clinical features and therapeutic outcomes. BMC Infect Dis. 2012;12:204.

5. Liang KL, Jiang RS, Lin JC, Chiu YJ, Shiao JY, Su MC, Hsin $\mathrm{CH}$. Central nervous system infection in patients with postirradiated nasopharyngeal carcinoma: a casecontrolled study. Am J Rhinol Allergy. 2009;23(4):417421.

6. Christopherson K, Werning JW, Malyapa RS, Morris CG, Mendenhall WM. Radiotherapy for sinonasal undifferentiated carcinoma. Am J Otolaryngol. 2014;35(2):141146.

7. Hayashi K, Koto M, Demizu Y, Saitoh JI, Suefuji H, Okimoto T, Ohno T, et al. A retrospective multicenter study of carbon-ion radiotherapy for major salivary gland carcinomas: Subanalysis of J-CROS 1402 HN. Cancer Sci. 2018;109(5):1576-1582.

8. Cheng KM, Chan CM, Fu YT, Ho LC, Tsang YW, Lee MK, Cheung YL, et al. Brain abscess formation in radiation necrosis of the temporal lobe following radiation therapy for nasopharyngeal carcinoma. Acta Neurochir (Wien). 2000;142(4):435-440; discussion 440-431.

9. Kuriyama H, Kajihara I, Kanemaru H, Nishimura Y, Igata $\mathrm{T}$, Masuguchi S, Imaoka Y, et al. Brain abscess in an angiosarcoma patient during a disease-free interval. Drug Discov Ther. 2019;13(3):175-176.

10. Noguchi T, Okada N, Tsuchiya Y, Sarukawa S, Fujita A, Nishino H, Jinbu Y, et al. Management of a brain abscess presumably caused by radiation osteomyelitis of the mandible. J Craniofac Surg. 2018;29(1):e18-e20.

11. Saito N, Nadgir RN, Nakahira M, Takahashi M, Uchino A, Kimura F, Truong MT, et al. Posttreatment CT and MR imaging in head and neck cancer: what the radiologist needs to know. Radiographics. 2012;32(5):1261-1282; discussion 1282-1264. 